\title{
Estimation of prevalence of sarcopenia by using a new bioelectrical impedance analysis in Chinese community-dwelling elderly people
}

\author{
Hui Wang ${ }^{1}$, Shan Hai', Li Cao', Jianghua Zhou², Ping Liư and Bi-Rong Dong ${ }^{1,3^{*}}$
}

\begin{abstract}
Background: The aim of the present study was to validate the usefulness of the new octapolar multifrequency bioelectrical impedance analysis (BIA) for assessment of appendicular skeletal muscle mass (ASM) by comparing it with that of dual-energy $X$-ray absorptiometry (DXA) and to investigate the prevalence of sarcopenia in Chinese community-dwelling elderly according to Asian Working Group for Sarcopenia (AWGS) definition.

Methods: A cross-sectional study was conducted in communities of Chengdu, China. A total of 944 communitydwelling elderly adults aged $\geq 60$ years were included. ASM was measured by using DXA as a criterion method to validate a standing eight-electrode multifrequency BIA (InBody 720), followed by a further estimation of the prevalence of sarcopenia according the AWGS definition.

Results: In the Bland-Altman analysis, no significant difference was found between DXA and BIA based on the ASM measurements. The prevalence of AWGS-defined sarcopenia was 12.5\% in the elderly women and $8.2 \%$ in the elderly men.

Conclusions: BIA is suitable for body composition monitoring (ASM) in elderly Chinese as a fast, noninvasive, and convenient method; therefore, it may be a better choice in large epidemiological studies in the Chinese population. The prevalence of AWGS-defined sarcopenia was approximately $10.4 \%$ and increased with age in the Chinese communitydwelling elderly in this study.
\end{abstract}

Keywords: Sarcopenia, Elderly, Dual-energy X-ray absorptiometry, Bioelectrical impedance analysis, Appendicular skeletal muscle, Asian Working Group for Sarcopenia

\section{Background}

Sarcopenia is the loss of muscle mass and function (defined by muscle strength or physical performance) with aging [1], which may result in reduced physical capability, quality of life, falls, disabilities, morbidities, and mortality, as well as high health care expenditure, in older people $[2,3]$. The prevalence of sarcopenia has been reported to increase rapidly with aging $[4,5]$. It was around $20 \%$ among people aged $\geq 65$ years and may reach $50-60 \%$ among octogenarians [6]. As China is

\footnotetext{
*Correspondence: birongdong@163.com

${ }^{1}$ Center of Gerontology and Geriatrics, West China Hospital, Sichuan University, No. 37 Guoxue Lane, Chengdu 610041, China

${ }^{3}$ Collaborative Innovation Center of Sichuan for Elderly Care and Health, Chengdu, China

Full list of author information is available at the end of the article
}

the most populated and fastest aging country in the world, sarcopenia poses a great impact on Chinese populations in the near future [7]. In 2010, the European Working Group on Sarcopenia in Older People (EWGSOP) proposed an operational definition and diagnostic strategy for sarcopenia that had become the most widely used in the world [8]. In 2014, the Asian Working Group for Sarcopenia (AWGS) also established the consensus on sarcopenia diagnosis, which recommended a set of approaches for the measurement of muscle mass, muscle strength, and physical performance, and chose different cutoff values according to the evidence derived from Asian populations. However, no epidemiological survey has used the AWGS definition to estimate the prevalence of sarcopenia in China. 
According to the AWGS definition, the measurable variables include muscle mass, muscle strength (handgrip strength $[\mathrm{HS}]$ ), and physical performance (gait speed [GS]). The challenge is to determine how best to measure them accurately, especially appendicular skeletal muscle mass (ASM). Several techniques have been used to assess ASM, including dual energy $x$-ray absorptiometry (DXA), computed tomography (CT), magnetic resonance imaging (MRI), and bioelectrical impedance analysis (BIA) [9]. Solid evidence from cadaver studies shows that DXA, MRI, and CT provide precise and reliable measurements of SM and thus can be considered as criterion methods for measuring ASM in vivo [10-12]. However, certain factors such as cost, accessibility, and the problem of radiation exposure limit the use of whole-body imaging. By contrast, BIA, which is based on measurements of tissue conductivity in the study of human body composition, is relatively simple, quick (takes only a few minutes), and noninvasive. Technological advances over the past decade include an increase in the number of contact electrodes from 4 to 8 [13, 14], and the use of multifrequency electrical levels to substitute the single-frequency BIA. By using low- and high-frequency electric currents, the multifrequency BIA methods successfully avoid the problems encountered in the use of primal BIA devices. The different currents allow for the estimation of extracellular and intracellular water, as well as tolal body water [15].

Previous studies verified that compared with the DXA method, multifrequency and eight-electrode BIA provides reliable measurements of body composition in healthy subjects and in patients with stable water levels $[16,17]$. InBody 720 (Biospace, Korea) is a new multifrequency analyzer with 4 pairs of electrodes (octapolar technology) embedded into the handles (thumb and palm electrodes) and floor scale (ball of foot and heel electrodes) of the analyzer. The researcher does not need to standardize the subject's posture before BIA, which has the potential to reduce measurement times and makes this instrument ideal for epidemiological studies. Some previous studies evaluated the accuracy of InBody 720 with different reference methods or in different populations [18-20], but InBody 720 has not been validated for the estimation of ASM in Chinese populations.

The first purpose of this study was to validate the usefulness of InBody 720 for ASM by comparing it with DXA. Its second purpose was to investigate the prevalence of sarcopenia according to the AWGS definition in Chinese community-dwelling elderly.

\section{Methods}

Design

A cross-sectional survey to determine the prevalence of sarcopenia according to the AWGS definition in a
Chinese community was conducted in two steps. First, 90 elderly volunteers (validation group) were recruited from 3 communities in Chengdu City to undergo BIA and DXA measurements to validate the accuracy of BIA for estimating ASM. Second, another 854 volunteers were recruited from the same communities with the same inclusive criterion, and the prevalence of sarcopenia in elderly people living in the community was investigated. The study staff was well trained in using investigation manuals, multimedia materials, and simulated patients. The trained interviewers collected the data from all the study participants at community centers by using face-toface interviews. In the validation group, the BIA and DXA measurements were performed within 1-3 days. All the subjects refrained from alcohol intake for at least $48 \mathrm{~h}$, from vigorous exercise for at least $12 \mathrm{~h}$, and from taking a meal or drink for at least $12 \mathrm{~h}$.

\section{Participants}

We recruited 944 subjects (462 men and 482 women) aged 60 to 92 years who voluntarily participated in the study, through leaflets and posters provided by the Center of Gerontology and Geriatrics, West China Hospital, Sichuan University, between March 2014 and October 2014. Volunteers were excluded if they had any diseases such as hyperthyroidism or hypothyroidism, or chronic heart and renal failure, or been receiving prescribed medications such as long-term steroid treatment, which is known to affect body composition. Individuals who could not communicate with the interviewers owing to severe cognitive impairment, mental disorders, and severe hearing and eye problems were also excluded from the study. All the participants were ambulatory without physical disability or amputation. The study was approved by the ethics committee of Sichuan University, under reference No. 2014 (57). Written consent was obtained from each participating subject prior to testing.

\section{Anthropometric measurements}

Each subject was interviewed by using a structured questionnaire to obtain basic demographic data and information on medical conditions. Weight and height were measured while barefoot and wearing light clothing. Body weight was measured to the nearest $0.1 \mathrm{~kg}$. Height was measured with a hypsometer to the nearest $0.1 \mathrm{~cm}$. Body mass index (BMI) was calculated as weight in kilograms divided by height in meters squared.

\section{Muscle strength and gait speed}

Muscle strength was assessed based on HS, measured by using a dynamometer (CAMRY EH101, CHINA). The participants were asked to exert maximum effort in a standing position, three readings were taken from each side, and the maximum value from the dominant hand 
was used for the analysis. Low handgrip strength was defined as $<26 \mathrm{~kg}$ for men and $<18 \mathrm{~kg}$ for women. The usual gait speed $(\mathrm{m} / \mathrm{s})$ on a $6-\mathrm{m}$ course was used as an objective measure of physical performance. The participants were asked to walk $6 \mathrm{~m}$ at their usual pace, and the time required to walk the distance was measured to calculate gait speed $(\mathrm{m} / \mathrm{s})$. Use of a cane or walker was permitted if the participants could not perform the gait test without it. The gait test was performed twice, and the mean value was used in the analysis. Slow walking speed was defined as a walking speed of $\leq 0.8 \mathrm{~m} / \mathrm{s}$.

\section{Dual-energy X-ray absorptiometry}

All the measurements were performed after overnight fasting and abstinence from alcohol and moderate to intensive exercise for more than $8 \mathrm{~h}$. The participants in validation group performed anthropometric measurements, DXA, and BIA on the same day. Each participant was scanned by using DXA (iDXA GE, USA) for measuring appendicular muscle mass. For the imaging examination, the participants wore cotton clothing without any metal attachments. Scans were performed in the whole-body scan mode in the following order: head, upper limbs, lower limbs, and trunk. Each participant underwent approximately $20 \mathrm{~min}$ of whole-body scan. Results were analyzed with software (12.10.017, GE, US).

\section{Bioelectrical impedance analysis}

Bioelectrical resistance was measured by using Inbody 720 (Biospace, Korea) at frequencies of 5, 50, 250, and $500 \mathrm{kHz}$. This instrument uses eight tactile electrodes, with four in contact with the palm and thumb of both hands and the other four in contact with the anterior and posterior aspects of the sole of both feet. The subject stands with the soles in contact with the foot electrodes and grabs the hand electrodes. The sequence of the measurements, controlled by a microprocessor, reports on the screen and paper. No precaution was taken to standardize the subject's posture before BIA, as suggested by the manufacturer. RI values were calculated at all frequencies. Data output, as calculated by using the manufacturer's algorithm, included fat mass and skeletal muscle mass of the total body, arms, and legs.

\section{Diagnosis of sarcopenia}

Sarcopenia was defined according to the AWGS algorithm, in which the patient has low muscle mass, and low muscle strength or low physical performance. As suggested by the AWGS, low muscle mass was defined as an ASM index (ASMI, ASM/height ${ }^{2}$ ) of $<7.0 \mathrm{~kg} / \mathrm{m}^{2}$ in men and $<5.7 \mathrm{~kg} / \mathrm{m}^{2}$ in women. Low muscle strength was defined as a handgrip strength of $<26 \mathrm{~kg}$ in men and $<18 \mathrm{~kg}$ in women; and low physical performance, as a gait speed of $<0.8 \mathrm{~m} / \mathrm{s}$.

\section{Statistical analyses}

All statistical analyses were performed by using SPSS version18.0 for Windows (IBM Corp, Armonk, NY). Baseline characteristics were compared between the men and women in the validated and elderly groups by using an independent-samples $t$ test for continuous variables and the Pearson chi-square test or Fisher exact test (for which an expected cell count was $<5$ ) for categorical variables. Pearson correlations were performed to examine the relationship between the DXA- and the InBody 720-measured ASMs. Bland-Altman analyses were performed to evaluate the extent of agreement between both methods. The differences in the continuous variables were compared by using one-way analysis of variance (ANOVA). Frequency data were compared by using the Pearson chi-square test or Fisher exact test (for which an expected cell count was $<5$ ). Differences were considered significant at $p<0.05$.

\section{Results}

\section{The characteristics of the subjects}

Table 1 lists the characteristics of the elderly participants according to sex. The mean age and BMI in the validated and elderly groups were respectively $68.71 \pm 6.60$ and $68.76 \pm 6.65$ years, and $24.27 \pm 3.25$ and $23.88 \pm 3.05$ $(p>0.1)$. We did not find significant sex-related differences in BMI and age. Although the weight and BMI in the elderly male group were smaller than those in the validated group $(p<0.05)$, the differences were not clinically significant. The sex-related differences in each anthropometric variable (expect BMI) were significant in both the validated and older groups. The men were taller and heavier, and had higher ASM and ASMI $(p<0.001)$. For physical performance, the men had significant stronger HS in both groups $(35.50 \pm 7.38$ vs. $23.29 \pm 4.12$ and $36.58 \pm$ 7.08 vs. $23.47 \pm 4.58$, respectively; $p<0.001$ ) and walked a little faster than the women in the elderly group (1.08 \pm 0.20 vs. $1.02 \pm 0.18, p<0.001)$. The percentages of current alcohol drinkers and smokers were 42.4 and $28.0 \%$ in the men, and 12.5 and $2.7 \%$ in the women, respectively. The most prevalent medical diseases were hypertension (43.5\%) and diabetes $(19.0 \%)$. No significant sex-related difference or differences between the validated and elderly groups were observed. The incidence rates of the remaining medical diseases were all $<5 \%$.

\section{Correlation between BIA- and DXA-measured ASM}

The correlation between ASM obtained by using BIA and DXA was high in both the men and women (Figs. 1a and 2a). The Pearson correlation coefficient and standard error of the estimate (SEE) of the regression equation were 0.94 and $1.05 \mathrm{~kg}$ in the men, and 0.90 and $0.93 \mathrm{~kg}$ in the women, respectively (both $p>0.05$ ). No significant method-related biases were found. The agreement of these two methods in 
Table 1 Comparison of baseline characteristics between the validated group and the elderly group

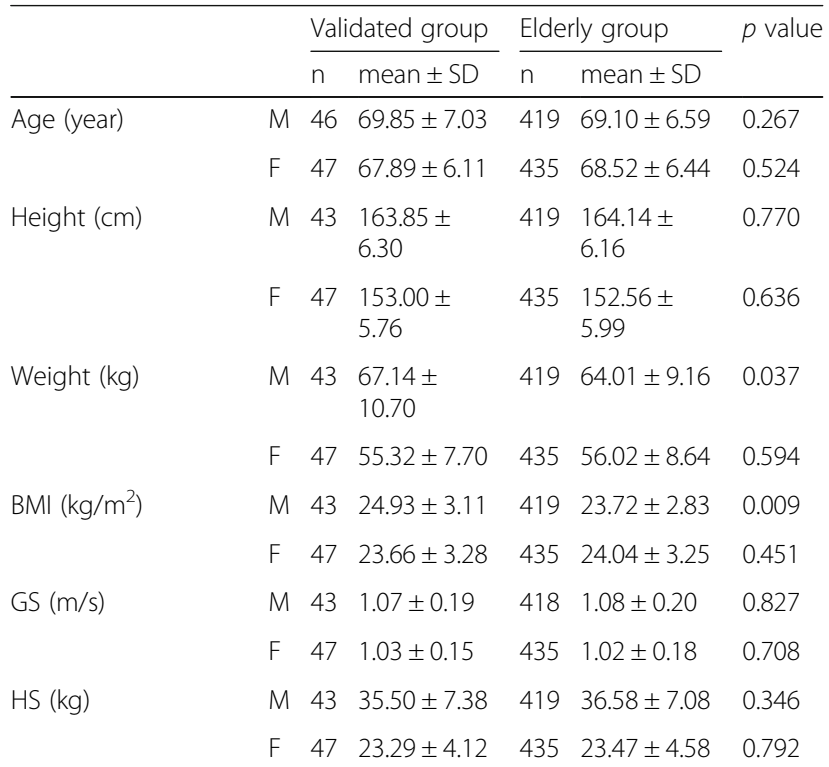

$\operatorname{ASM}(\mathrm{kg})$

BIA

DEXA

$\begin{array}{lllll}\text { M } 43 & 19.72 \pm 2.85 & 419 & 19.63 \pm 2.95 & 0.830\end{array}$

F $\quad 47 \quad 13.86 \pm 2.25 \quad 435 \quad 14.02 \pm 2.36 \quad 0.667$

M $43 \quad 18.74 \pm 3.16 \quad 0 \quad *$

F $47 \quad 13.37 \pm 2.13 \quad 0 \quad *$

ASMI $\left(\mathrm{kg} / \mathrm{m}^{2}\right)$

BIA

DEXA

Medical diagnoses

Hypertension

M $43 \quad 19(44.2)$

$419 \quad 178(42.5)$

0.830

F $47 \quad 18(38.3)$

$435 \quad 196(45.2)$

0.368

Diabetes

M 436 (14.0)

$41982(19.6)$

0.372

F $47 \quad 10(21.3)$

43582 (18.9)

0.688

Thyroid diseases

M 430

$4192(0.5)$

1.000

F 470

Cardiac diseases

M 431 (2.3)

F $47 \quad 7(14.9)$

$435 \quad 4(0.9)$

1.000

$419 \quad 23(5.5)$

0.373

$435 \quad 20(4.6)$

Renal diseases

M 430

$4196(1.4)$

$435 \quad 7(1.6)$

1.000

F $47 \quad 2$ (4.3)

Stroke

M 430

F 470

Cancer

COPD

M $43 \quad 1(2.3)$

F 470

M 430

F $47 \quad 1(2.1)$

$418 \quad 4(1.0)$

$434 \quad 5(1.2)$

$4193(0.7)$

$4352(0.5)$

4195 (1.2)

$4357(1.6)$

Tuberculosis
M 430
Table 1 Comparison of baseline characteristics between the validated group and the elderly group (Continued)

\begin{tabular}{lllllll}
\hline & $F$ & 47 & 0 & 435 & $1(0.2)$ & 1.000 \\
Hepatic diseases & $M$ & 43 & $1(2.3)$ & 419 & $8(1.9)$ & 0.588 \\
& $F$ & 47 & 0 & 435 & $7(1.6)$ & 1.000 \\
Smoking habits & $M$ & 43 & $12(27.9)$ & 419 & $118(28.2)$ & 0.972 \\
& F & 47 & 0 & 435 & $13(3.0)$ & 0.626 \\
Alcohol & $M$ & 43 & $24(55.8)$ & 419 & $173(41.3)$ & 0.067 \\
consumption & & & & & & \\
& $F$ & 47 & $12(25.5)$ & 435 & $48(11.1)$ & 0.004
\end{tabular}

*: no data; BMI body mass index, GS gait speed, HS handgrip strength, ASM appendicular skeletal muscle mass, ASMI appendicular skeletal muscle mass index, BIA bioelectrical impedance analysis, DXA dual energy x-ray absorptiometry, COPD chronic obstructive pulmonary disease; Using independent-samples $t$ test for continuous variables and Pearson chi-square or Fisher exact test (where an expected cell count was $<5$ ) for categorical variables. During testing, $p<0.05$ was considered statistically significant

the Bland-Altman analysis is presented in Figs. $1 \mathrm{~b}$ and $2 \mathrm{~b}$. Only 2 points were outside the limit of agreement in the men; and 3 points, in the women.

Differences in body composition, muscle strength, and physical function according to age and sex

The differences in body composition, physical functions (represented by GS), and muscle strength (represented by HS) according to age and sex are shown in Table 2. ASM, ASMI, GS, and HS all showed significant declines with age in both sexes $(p<0.005)$.

\section{Estimated prevalence of low SM, low HS, low GS, and sarcopenia in the different age groups and the elderly group}

The estimated prevalence of low SM, low HS, low GS, and sarcopenia in the different age groups and the elderly group are shown in Table 3. The criteria, including low SM, low HS, low GS, and sarcopenia, were defined by the AWGS [2]. The prevalence rates of low SM, low HS, and low GS increased significantly with age $(p<0.001)$. When the AWGS criteria were used to define sarcopenia, the prevalence rates of sarcopenia were $2.3,5.6$, and $19.9 \%$ in all the participants aged 60-64, 65-74, and $\geq 75$ years, respectively $(p<0.001)$, and the age-related trend was also apparent in both sexes $(p<0.001)$. The prevalence rates of sarcopenia were $8.2,12.5$, and $10.4 \%$ in the elderly women, men, and both sexes, respectively.

\section{Discussion}

MRI, CT, and DXA measurements of ASM are not suitable for large-scale surveys on sarcopenia in the elderly due to cost, accessibility, and the problem of radiation exposure. BIA might be a good alternative method to measure ASM for epidemiological surveys. However, a previous study reported that BIA equations chosen should not be used without prior verification against reference methods in the 


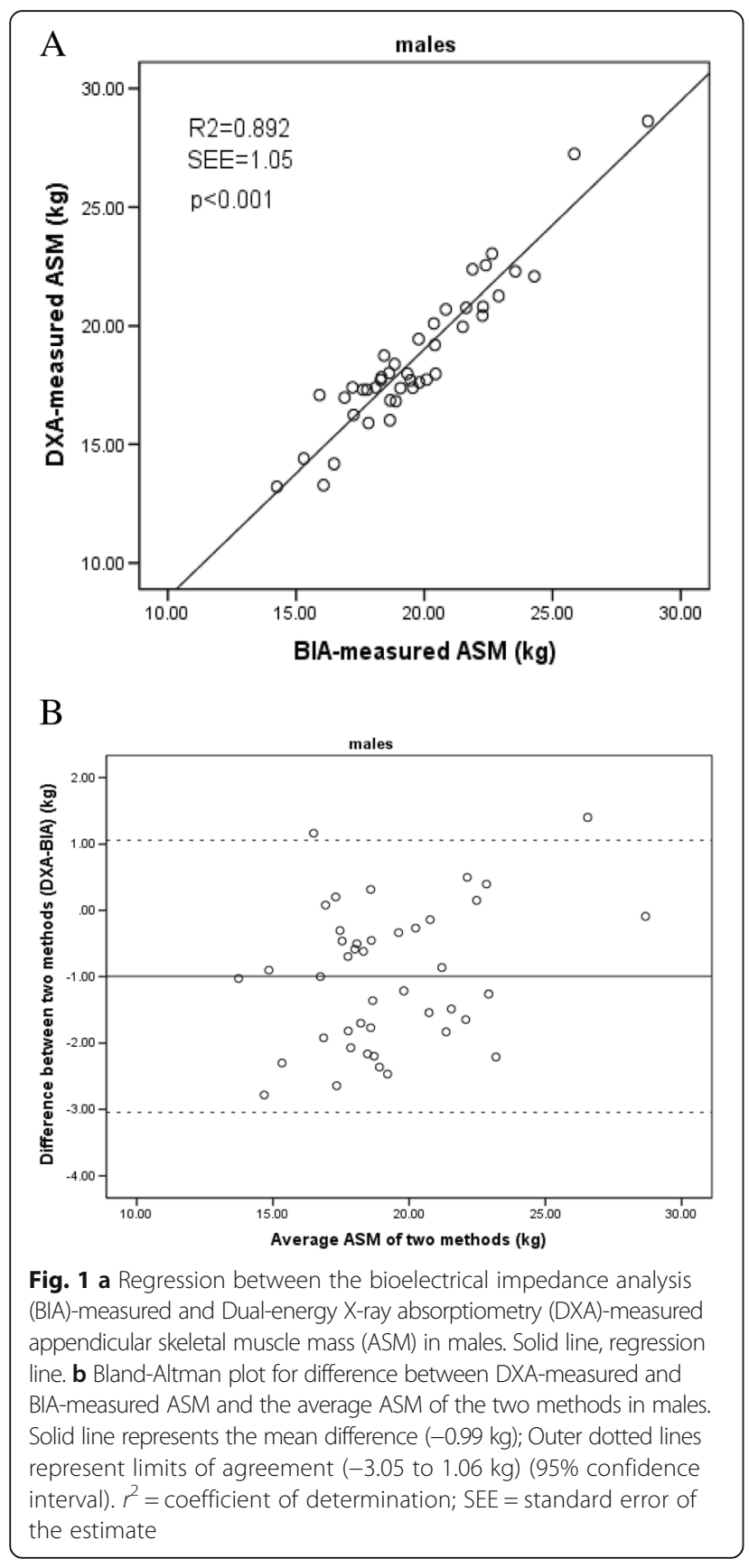

subject population studied because of existing differences in body build among ethnic groups [21]. To the best of our knowledge, this is the first study that validated the usefulness of a multifrequency octapolar BIA, InBody 720, and its predictive ability for estimating ASM by comparing it with those of DXA in community-dwelling elderly people in China. The results of the present study demonstrated the Pearson correlation coefficients $(0.944$ and 0.903$)$ and SEE $(1.051$ and $0.927 \mathrm{~kg})$ in men and women, respectively, in predicting ASM, which were similar to those obtained in previous reports $[16,22,23]$. Houtkouper et al. suggested

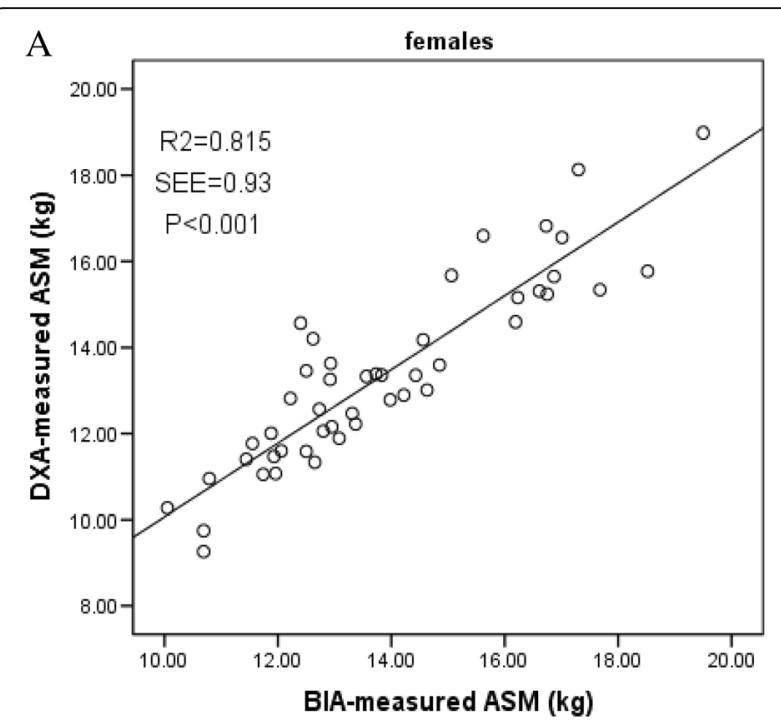

B

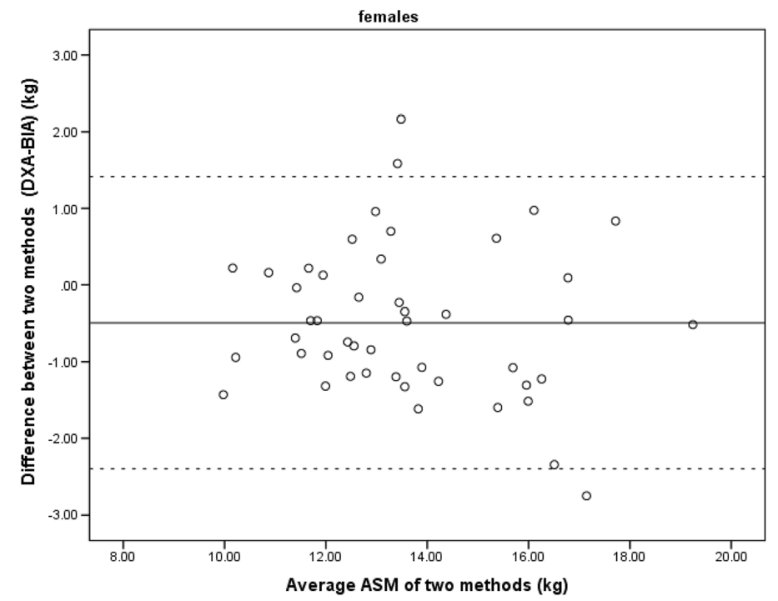

Fig. 2 a Regression between the bioelectrical impedance analysis (BIA)-measured and Dual-energy X-ray absorptiometry (DXA)-measured appendicular skeletal muscle mass (ASM) in females. Solid line, regression line. $\mathbf{b}$ Bland-Altman plot for difference between DXA-measured and BIA-measured ASM and the average ASM of the two methods in females. Solid line represents the mean difference $(-0.49 \mathrm{~kg})$; Outer dotted lines represent limits of agreement ( -2.40 to $1.41 \mathrm{~kg})(95 \%$ confidence interval). $r^{2}=$ coefficient of determination; $\mathrm{SEE}=$ standard error of the estimate

that prediction errors (SEE) of $2.0-2.5 \mathrm{~kg}$ in men and $1.5-1.8 \mathrm{~kg}$ in women are considered ideal [21]. Although the negative slope of the Bland-Altman plot indicated a trend of underestimation and overestimation errors with the BIA method for participants with lower and higher ASMs, respectively, the mean error was small and insignificant. Thus, this result suggests that the BIA method provided a meaningful ASM estimation in the Chinese populations. However, our observation conflicts with the results of Anderson et al., who found a significant InBody 720 underestimation of ASM in men $(-3.0 \mathrm{~kg})$ 
Table 2 Differences in body composition, muscle strength and physical function with age and gender

\begin{tabular}{lllllll}
\hline & Age group (year) & Number & ASM (kg) & ASMl (kg/m2) & HS (kg) & GS (s) \\
\hline Males & $60-64$ & 146 & $20.24 \pm 0.26$ & $7.43 \pm 0.06$ & $39.62 \pm 0.54$ & $1.12 \pm 0.02$ \\
& $65-74$ & 200 & $19.55 \pm 0.20$ & $7.23 \pm 0.05$ & $36.64 \pm 0.46$ & $1.09 \pm 0.01$ \\
& $\geq 75$ & 117 & $19.03 \pm 0.27$ & $7.09 \pm 0.07$ & $32.21 \pm 0.63$ & $0.99 \pm 0.02$ \\
$p$ & & 0.003 & 0.001 & $<0.001$ & $<0.001$ \\
Females & $60-64$ & 162 & $14.55 \pm 0.18$ & $6.10 \pm 0.06$ & $25.06 \pm 0.35$ & $1.08 \pm 0.01$ \\
& $65-74$ & 226 & $14.13 \pm 0.15$ & $6.03 \pm 0.05$ & $23.40 \pm 0.28$ & $1.02 \pm 0.01$ \\
& $\geq 75$ & 94 & $12.76 \pm 0.22$ & $5.67 \pm 0.07$ & $20.80 \pm 0.44$ & $0.92 \pm 0.02$ \\
$p$ & & & $<0.001$ & $<0.001$ & $<0.001$ & $<0.001$ \\
\hline
\end{tabular}

ASM appendicular skeletal muscle mass, ASMI appendicular skeletal muscle mass index, HS handgrip strength, GS gait speed, Using one-way ANOVA for continuous variables. During testing, $p<0.05$ was considered statistically significant

and women $(-1.0 \mathrm{~kg})[24]$. Their study population was quite different from ours; their subjects were not Asians, were aged 18 to 49 years, and had slightly higher BMIs $\left(25.8 \pm 4.5 \mathrm{~kg} / \mathrm{m}^{2}\right)$. Another study conducted in a Finnish population found that compared with DXA, InBody 720 provided on average $2-6 \%$ lower values for fat mass (FM) in men with normal BMI and in all BMI categories in women. However, the authors did not report the correlation of ASM. But in our recent research, we did not validate the correlation according to BMI categories.

Association of sarcopenia with poor health status and adverse outcomes had triggered a new approach for health promotion and health care of older people. Nutrition and exercise interventions can help prevent age-related decline in physical performance and increase in mortality $[25,26]$. Although the prevalence of sarcopenia in elderly people has been broadly investigated in Asia, the large variability in prevalence is partly related to the differences in measurements and cutoffs used to define sarcopenia. Hence, the AWGS developed a consensus definition and cutoff points for sarcopenia in Asia. To our knowledge, this is the first large epidemiological survey that used the AWGS definition to estimate the prevalence of sarcopenia in Chinese community-dwelling elderly. The results show prevalence rates of $12.5 \%$ and $8.2 \%$ in women and men, respectively. The prevalence of sarcopenia varies across studies because of different study populations, ages, sex, and diagnosis criteria [25]. Focusing on the Chinese elderly, between-study variations in the prevalence of sarcopenia also exists. We found only 3 studies that used the AWGS definition in Chinese elderly. One study is currently conducted in Chinese suburb-dwelling elderly, and the authors reported that the prevalence of sarcopenia was $11.5 \%$ in women and $6.4 \%$ in men [27], which were similar to the outcomes in our study. Another research study found that the prevalence rates of sarcopenia (using the AWGS definition) were $5.9 \%$ in men and $0.7 \%$ in women [18], which were much lower than ours. Their research only included 286 participants with a mean age of $66.5 \pm 4.8$ years in men and $65.4 \pm 4.5$ years in women. The difference in age partly resulted in the diversity of prevalence. Another study reported that the prevalence rates of AWGS-defined sarcopenia were 7.0 and $13.1 \%$ in Chinese urban and rural elderly, respectively. These values indicate a significant difference, which was partly due

Table 3 Estimated prevalence of low SM, low HS, low GS and sarcopenia in different age groups and the elderly

\begin{tabular}{|c|c|c|c|c|c|c|}
\hline & Age group (year) & Number & Low muscle mass & Low gait speed & Low handgrip strenth & Sarcopenia \\
\hline \multirow[t]{4}{*}{ Male } & $60-64$ & 146 & $40(27.4)$ & $4(2.7)$ & $5(3.4)$ & $4(2.7)$ \\
\hline & $65-74$ & 199 & $68(34.2)$ & $9(4.5)$ & $4(2.0)$ & $8(4.0)$ \\
\hline & $\geq 75$ & 117 & $52(44.4)$ & $18(15.4)$ & $20(17.1)$ & $18(15.4)$ \\
\hline & elderly & 316 & $120(38.0)$ & $27(8.6)$ & $24(7.6)$ & $26(8.2)$ \\
\hline$p$ & & & 0.015 & $<0.001$ & $<0.001$ & $<0.001$ \\
\hline \multirow[t]{4}{*}{ Female } & $60-64$ & 162 & $20(12.3)$ & $6(3.7)$ & $6(3.7)$ & $3(1.9)$ \\
\hline & $65-74$ & 226 & $37(16.4)$ & $17(7.5)$ & $17(7.5)$ & $16(7.1)$ \\
\hline & $\geq 75$ & 94 & $36(38.3)$ & $22(23.4)$ & $25(26.2)$ & $24(25.5)$ \\
\hline & elderly & 320 & $73(22.8)$ & $39(12.2)$ & $42(13.1)$ & $40(12.5)$ \\
\hline$p$ & & & $<0.001$ & $<0.001$ & $<0.001$ & $<0.001$ \\
\hline
\end{tabular}

Low muscle mass was defined as appendicular skeletal muscle mass index (ASMI) $<7 \mathrm{~kg} / \mathrm{m} 2$ for males and $<5.7 \mathrm{~kg} / \mathrm{m} 2$ for females; low gait speed (GS) as GS $<0.8 \mathrm{~m} / \mathrm{s}$; low handgrip strength (HS) as HS $<26 \mathrm{~kg}$ for males or $<18 \mathrm{~kg}$ for females. Using Pearson chi-Square tests or Fisher exact test (for which an expected cell count was $<5$ ) for categorical variables. Comparison between $60-64,65-74$, and $\geq 75, p<0.05$ was considered statistically significant 
to underdevelopment of living and socioeconomic conditions in rural areas. However, the researchers applied only anthropometric measures for estimating skeletal muscle mass instead of BIA or DXA [28]. Some studies also investigated the prevalence of sarcopenia in Chinese populations by using different diagnostic criteria. According to a large cohort study involving 4000 Chinese communitydwelling adults aged $\geq 65$ years in Hong Kong, the prevalence of sarcopenia (defined by the EWGSOP criteria) was $9 \%$ at baseline [29], which was similar to the outcome in our study. However, a survey in Taiwan found that the prevalence (defined by a BMI of $8.87 \mathrm{~kg} / \mathrm{m}^{2}$ in men and $6.42 \mathrm{~kg} / \mathrm{m}^{2}$ in women) of sarcopenia was $18.6 \%$ in women and $23.6 \%$ in men, which were much higher than our results. It also reported a great discrepancy in prevalence of sarcopenia, from 14 to $37 \%$ in women and from 13 to $42 \%$ in men, in the same sample depending on the definition used [5]. The tremendous variations within the same population shown in the previous research studies bring into question the results of the existing studies on sarcopenia prevalence. These variations were partly due to the different participants and definitions of sarcopenia. Furthermore, the appropriate definition of sarcopenia in Chinese older adults remains unclear [30,31]. Therefore, large population-based perspective surveys are required for Chinese people to establish ethnic-specific references and cutoff values for older adults [32].

Similar to those of other authors [4, 30, 33, 34], our present findings also confirmed the decrease in ASM, ASMI, HS, and GS, and the increase in the prevalence rates of low muscle mass, low muscle strength, and low physical performance with age. We observed that ASM was significantly lower among the women aged $\geq 75$ years than among those in the preceding age group (12.76 \pm $0.22 \mathrm{~kg}$ vs. $14.13 \pm 0.15 \mathrm{~kg}, p<0.001)$. Moreover, even though ASM decreased dramatically, body weight just decreased slightly with aging, partly due to the increase in FM (unreported data). The decrease in ASM and increase in FM accompanying aging may lead to the development of sarcopenia and contribute to obesity in the elderly [33]. We also found that muscle strength (HS) and physical performance (GS) decreased more greatly with age than did muscle mass $(p<0.001)$, which was similar to the findings of previous cross-sectional or longitudinal investigations [30, 35-38]. Goodpaster found that even maintenance or gain of lean mass in the elderly did not prevent loss of strength [39]. Despite extensive research efforts, the mechanism responsible for the age-associated decline in muscle strength has not been completely elucidated, but the age-associated decline in muscle mass certainly plays a partial role [38]. Muscle strength, rather than muscle mass, is more important in estimating adverse outcomes such as physical disability, poor quality of life, and higher mortality [39-41]. For this reason, both EWGS and AWGS recommended the use of both low muscle mass and function (strength or performance) for the diagnosis of sarcopenia $[2,8]$.

\section{Study limitations}

Some limitations of this study should be pointed out. First, as previously mentioned, nonrandom selection of the study population and the exclusion of elderly people with severe chronic diseases from our study group might have resulted in a selection bias. Consequently, the study group, which included individuals who were relatively healthy and had good activity level, might have caused, to some extent, the underestimation of the prevalence of sarcopenia. Second, we used DXA as the "gold standard" reference method to validate BIA (InBody 720). Although validation in comparison with DXA is the most feasible and appropriate choice for our research, it is possibly not the most accurate analysis. The sample sizes for the Bland-Altman analysis should be more than 100; however, in our study, we only collected 90 participants because of the limited condition. In our future research, we should expand the sample sizes to decrease the bias. Finally, as a cross-sectional study, it may not provide the same results as a longitudinal or semi-longitudinal study. This paper presents the first-stage research results. In the next-stage research, the change trend of muscle mass, muscle strength, physical performance, and other outcome indicators such as falls, hospital admission, and mortality will be investigated.

\section{Conclusion}

In conclusion, the relatively high correlation coefficient between the BIA- (InBody 720) and DXA-measured ASMs indicates that the application is suitable for body composition monitoring in elderly Chinese in a fast, noninvasive, and convenient way. The study also demonstrates that the prevalence of AWGS-defined sarcopenia was approximately $10.4 \%$ and increased with age in a sample of Chinese community-dwelling elderly.

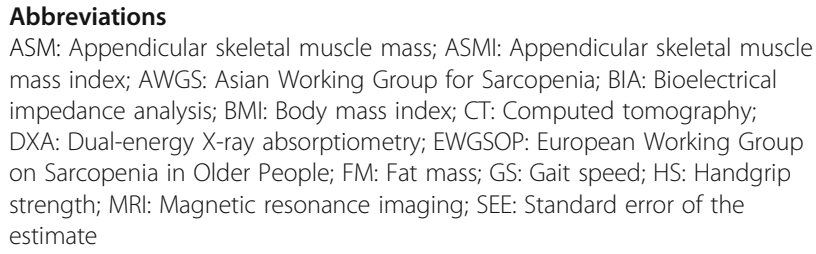
mass index; AWGS: Asian Working Group for Sarcopenia; BIA: Bioelectrical impedance analysis; BMI: Body mass index; CT: Computed tomography; DXA: Dual-energy X-ray absorptiometry; EWGSOP: European Working Group on Sarcopenia in Older People; FM: Fat mass; GS: Gait speed; HS: Handgrip strength; MRI: Magnetic resonance imaging; SEE: Standard error of the estimate

\section{Acknowledgments}

The authors thank the staff of the Department of Geriatrics Medicine, West China Hospital, for their guidance and support. We also thank the employees of Yulin Community Hospital for providing free space.

\section{Funding}

This research was supported by a project grant from the Science and Technology Project of Sichuan Province (2014SZ0153). The sponsor had no role in the design, methods, data collection, analysis, and preparation of this paper. 


\section{Availability of data and materials}

The datasets during and/or analysis during the present study are available from the corresponding author on reasonable request.

\section{Authors' contributions}

$B R D, H W, S H$, and $L C$ designed the study. HW, SH, LC, JHZ, and YY recruited the subjects and collected the data. HW and PL performed the statistical analyses. HW drafted the manuscript. All the authors commented on the drafts and approved the final draft. HW is the guarantor of the manuscript.

\section{Competing interests}

The authors declare that they have no competing interests.

\section{Consent for publication}

Not applicable.

\section{Ethics approval and consent to participate}

The study was approved by the ethics committee of Sichuan University, under reference No. 2014 (57). Written consent was obtained from each participating subject prior to testing.

\section{Author details}

${ }^{1}$ Center of Gerontology and Geriatrics, West China Hospital, Sichuan University, No. 37 Guoxue Lane, Chengdu 610041, China. ${ }^{2}$ School of Clinical Medicine, Chengdu Medical College, Chengdu, China. ${ }^{3}$ Collaborative Innovation Center of Sichuan for Elderly Care and Health, Chengdu, China.

\section{Received: 21 July 2016 Accepted: 1 December 2016}

\section{Published online: 28 December 2016}

\section{References}

1. Delmonico MJ, Harris TB, Visser M, Park SW, Conroy MB, Velasquez-Mieyer P, Boudreau R, Manini TM, Nevitt M, Newman AB, et al. Longitudinal study of muscle strength, quality, and adipose tissue infiltration. Am J Clin Nutr. 2009;90(6):1579-85.

2. Chen LK, Liu LK, Woo J, Assantachai P, Auyeung TW, Bahyah KS, Chou MY, Chen LY, Hsu PS, Krairit O, et al. Sarcopenia in Asia: consensus report of the Asian Working Group for Sarcopenia. J Am Med Dir Assoc. 2014;15(2):95-101.

3. Chuang SY, Chang HY, Lee MS, Chia-Yu Chen R, Pan WH. Skeletal muscle mass and risk of death in an elderly population. Nutr Metab Cardiovasc Dis. 2014;24(7):784-91.

4. lannuzzi-Sucich M, Prestwood KM, Kenny AM. Prevalence of sarcopenia and predictors of skeletal muscle mass in healthy, older men and women. J Gerontol A Biol Sci Med Sci. 2002;57(12):M772-7.

5. Chien MY, Huang TY, Wu YT. Prevalence of sarcopenia estimated using a bioelectrical impedance analysis prediction equation in communitydwelling elderly people in Taiwan. J Am Geriatr Soc. 2008;56(9):1710-5.

6. Morley JE. Sarcopenia: diagnosis and treatment. J Nutr Health Aging. 2008; 12(7):452-6.

7. Woo J. Meeting the needs of an aging population in China: Public health and policy implications. J Clin Gerontol Geriatr. 2013;4(2):31-2.

8. Cruz-Jentoft AJ, Baeyens JP, Bauer JM, Boirie Y, Cederholm T, Landi F, Martin FC, Michel JP, Rolland Y, Schneider SM, et al. Sarcopenia: European consensus on definition and diagnosis: Report of the European Working Group on Sarcopenia in Older People. Age Ageing. 2010;39(4):412-23.

9. Heymsfield SB, Adamek M, Gonzalez MC, Jia G, Thomas DM. Assessing skeletal muscle mass: historical overview and state of the art. J Cachex Sarcopenia Muscle. 2014;5(1):9-18.

10. Mitsiopoulos N, Baumgartner RN, Heymsfield SB, Lyons W, Gallagher D, Ross R. Cadaver validation of skeletal muscle measurement by magnetic resonance imaging and computerized tomography. J Appl Physiol (1985). 1998;85(1):115-22.

11. Lee RC, Wang ZM, Heymsfield SB. Skeletal muscle mass and aging: regional and whole-body measurement methods. Can J Appl Physiol. 2001;26(1):102-22.

12. Heymsfield SB, Gallagher D, Visser M, Nunez C, Wang ZM. Measurement of skeletal muscle: laboratory and epidemiological methods. J Gerontol A Biol Sci Med Sci. 1995;50 Spec No:23-9.

13. Tan YX, Nunez C, Sun Y, Zhang K, Wang Z, Heymsfield SB. New electrode system for rapid whole-body and segmental bioimpedance assessment. Med Sci Sports Exerc. 1997;29(9):1269-73.
14. Nunez C, Gallagher D, Visser M, Pi-Sunyer FX, Wang Z, Heymsfield SB. Bioimpedance analysis: evaluation of leg-to-leg system based on pressure contact footpad electrodes. Med Sci Sports Exerc. 1997;29(4):524-31.

15. Baumgartner RN, Stauber PM, Koehler KM, Romero L, Garry PJ. Associations of fat and muscle masses with bone mineral in elderly men and women. Am J Clin Nutr. 1996;63(3):365-72.

16. Pietrobelli A, Rubiano F, St-Onge MP, Heymsfield SB. New bioimpedance analysis system: improved phenotyping with whole-body analysis. Eur J Clin Nutr. 2004;58(11):1479-84.

17. Neovius $M$, Hemmingsson E, Freyschuss B, Udden J. Bioelectrical impedance underestimates total and truncal fatness in abdominally obese women. Obesity (Silver Spring). 2006;14(10):1731-8.

18. Gibson AL, Holmes JC, Desautels RL, Edmonds LB, Nuudi L. Ability of new octapolar bioimpedance spectroscopy analyzers to predict 4-componentmodel percentage body fat in Hispanic, black, and white adults. Am J Clin Nutr. 2008;87(2):332-8

19. Volgyi E, Tylavsky FA, Lyytikainen A, Suominen H, Alen M, Cheng SL. Assessing body composition with DXA and bioimpedance: effects of obesity, physical activity, and age. Obesity. 2008;16(3):700-5.

20. Bedogni G, Malavolti M, Severi S, Poli M, Mussi C, Fantuzzi AL, Battistini N. Accuracy of an eight-point tactile-electrode impedance method in the assessment of total body water. Eur J Clin Nutr. 2002;56(11):1143-8.

21. Houtkooper LB, Lohman TG, Going SB, Howell WH. Why bioelectrical impedance analysis should be used for estimating adiposity. Am J Clin Nutr. 1996;64(3):436-48.

22. Kyle UG, Genton L, Hans D, Pichard C. Validation of a bioelectrical impedance analysis equation to predict appendicular skeletal muscle mass (ASMM). Clin Nutr. 2003;22(6):537-43.

23. Salinari S, Bertuzzi A, Mingrone G, Capristo E, Scarfone A, Greco AV, Heymsfield SB. Bioimpedance analysis: a useful technique for assessing appendicular lean soft tissue mass and distribution. J Appl Physiol (1985). 2003;94(4):1552-6.

24. Anderson LJ, Erceg DN, Schroeder ET. Utility of multifrequency bioelectrical impedance compared with dual-energy x-ray absorptiometry for assessment of total and regional body composition varies between men and women. Nutr Res. 2012;32(7):479-85.

25. Cruz-Jentoft AJ, Landi F, Schneider SM, Zuniga C, Arai H, Boirie Y, Chen LK, Fielding RA, Martin FC, Michel JP, et al. Prevalence of and interventions for sarcopenia in ageing adults: a systematic review. Report of the International Sarcopenia Initiative (EWGSOP and IWGS). Age Ageing. 2014;43(6):748-59.

26. Harber MP, Konopka AR, Douglass MD, Minchev K, Kaminsky LA, Trappe TA, Trappe S. Aerobic exercise training improves whole muscle and single myofiber size and function in older women. Am J Physiol Regul Integr Comp Physiol. 2009;297(5):R1452-9.

27. Han P, Kang L, Guo Q, Wang J, Zhang W, Shen S, Wang X, Dong R, Ma Y, Shi $Y$, et al. Prevalence and factors associated with sarcopenia in suburbdwelling older Chinese using the Asian Working Group for Sarcopenia Definition. J Gerontol A Biol Sci Med Sci. 2016;71(4):529-35.

28. Gao LL, Jiang JJ, Yang M, Hao QK, Luo L, Dong BR. Prevalence of sarcopenia and associated factors in Chinese community-dwelling elderly: comparison between rural and urban areas. J Am Med Dir Assoc. 2015;16(11):1003.e1-6.

29. Yu R, Wong M, Leung J, Lee J, Auyeung TW, Woo J. Incidence, reversibility, risk factors and the protective effect of high body mass index against sarcopenia in community-dwelling older Chinese adults. Geriatr Gerontol Int. 2014;14 Suppl 1:15-28.

30. Zeng P, Wu SN, Han YW, Liu JM, Zhang Y, Zhang EY, Zhang Y, Gong H, Pang J, Tang ZL, et al. Differences in body composition and physical functions associated with sarcopenia in Chinese elderly: Reference values and prevalence. Arch Gerontol Geriatr. 2015;60(1):118-23.

31. Wen $X$, Wang $M$, Jiang CM, Zhang YM. Are current definitions of sarcopenia applicable for older Chinese adults? J Nutr Health Aging. 2011;15(10):847-51.

32. Kim H, Hirano H, Edahiro A, Ohara Y, Watanabe Y, Kojima N, Kim M, Hosoi E, Yoshida $Y$, Yoshida $H$, et al. Sarcopenia: prevalence and associated factors based on different suggested definitions in community-dwelling older adults. Geriatr Gerontol Int. 2016;16:110-22.

33. Gaba A, Pridalova M. Age-related changes in body composition in a sample of Czech women aged 18-89 years: a cross-sectional study. Eur J Nutr. 2014; 53(1):167-76.

34. Coin A, Sergi G, Minicuci N, Giannini S, Barbiero E, Manzato E, Pedrazzoni M, Minisola S, Rossini M, Del Puente A, et al. Fat-free mass and fat mass reference values by dual-energy X-ray absorptiometry (DEXA) in a 20-80 year-old Italian population. Clin Nutr. 2008;27(1):87-94. 
35. Frontera WR, Hughes VA, Fielding RA, Fiatarone MA, Evans WJ, Roubenoff R. Aging of skeletal muscle: a 12-yr longitudinal study. J Appl Physiol (1985). 2002;88(4):1321

36. Rantanen T, Masaki K, Foley D, Izmirlian G, White L, Guralnik JM. Grip strength changes over $27 \mathrm{yr}$ in Japanese-American men. J Appl Physiol (1985). 1998;85(6):2047-53.

37. Schiller BC, Casas YG, Tracy BL, DeSouza CA, Seals DR. Age-related declines in knee extensor strength and physical performance in healthy Hispanic and Caucasian women. J Gerontol A Biol Sci Med Sci. 2000;55(12):B563-9.

38. Lauretani F, Russo CR, Bandinelli S, Bartali B, Cavazzini C, Di lorio A, Corsi AM, Rantanen T, Guralnik JM, Ferrucci L. Age associated changes in skeletal muscles and their effect on mobility: an operational diagnosis of sarcopenia. J Appl Physiol (1985). 2003;95(5):1851-60.

39. Goodpaster BH, Park SW, Harris TB, Kritchevsky SB, Nevitt M, Schwartz AV, Simonsick EM, Tylavsky FA, Visser M, Newman AB, et al. The loss of skeletal muscle strength, mass, and quality in older adults: The health, aging and body composition study. J GerontolAa Biol Sci Med Sci. 2006;61 (10):1059-64.

40. Delmonico MJ, Harris TB, Lee JS, Visser M, Nevitt M, Kritchevsky SB, Tylavsky FA, Newman AB, Health A, Body Composition S. Alternative definitions of sarcopenia, lower extremity performance, and functional impairment with aging in older men and women. J Am Geriatr Soc. 2007;55(5):769-74.

41. Newman AB, Kupelian V, Visser M, Simonsick EM, Goodpaster BH, Kritchevsky SB, Tylavsky FA, Rubin SM, Harris TB. Strength, but not muscle mass, is associated with mortality in the health, aging and body composition study cohort. J Gerontol A Biol Sci Med Sci. 2006;61(1):72-7.

\section{Submit your next manuscript to BioMed Central and we will help you at every step:}

- We accept pre-submission inquiries

- Our selector tool helps you to find the most relevant journal

- We provide round the clock customer support

- Convenient online submission

- Thorough peer review

- Inclusion in PubMed and all major indexing services

- Maximum visibility for your research

Submit your manuscript at www.biomedcentral.com/submit 